\title{
ESTUDO CLÍNICO-PATOLÓGICO, DA PROLIFERAÇÃO CELULAR E DA APOPTOSE NO ADENOCARCINOMA GÁSTRICO DA CÁRDIA
}

\author{
Ana Maria Amaral Antonio MADER ${ }^{1}$, Francy Reis da Silva PATRÍCIO' ${ }^{1}$ \\ Moacyr Pezati RIGUEIRO ${ }^{1}$ e Laercio Gomes LOURENÇO ${ }^{2}$
}

RESUMO - Racional/Objetivo - Em vista do aumento na incidência do carcinoma da cárdia nos últimos anos, este trabalho visa estudar os aspectos clínico-patológicos, da proliferação celular e da apoptose tumorais, suas correlações e eventuais influências no seu prognóstico. Material e Método - Estudaram-se 40 doentes submetidos a gastrectomia total por carcinoma da cárdia, no período de 1988 a 2001, com seguimento clínico de no mínimo 3 anos. Excluíram-se aqueles com tratamento químio ou radioterápico prévio, neoplasia precoce, óbito no intra-operatório ou por outras causas não relacionadas ao câncer. Analisou-se sexo, idade, tipo histológico de Laurén, padrão de crescimento tumoral de Ming, estádio e presença ou ausência de metaplasia intestinal e/ou Helicobacter pylori na mucosa adjacente. O índice apoptótico foi avaliado por cortes histológicos corados pela hematoxilina-eosina. $\mathrm{O}$ índice de proliferação celular foi avaliado por meio da imunoexpressão ao PCNA, sendo a técnica imunoistoquímica utilizada a da estreptoavidina-biotina-peroxidase. Para análise da sobrevida, excluíram-se os casos com metástase à distância ao diagnóstico. Foram utilizados testes $t$ de Student, de Mann-Whitney, curvas de Kaplan-Meier e modelo de regressão de Cox. O nível de significância adotado foi menor de 0,05. Resultados - A idade média foi de 61 anos (mediana: 63); houve predomínio do sexo masculino (72,5\%), tipo histológico difuso (55\%) e infiltrativo (72,5\%) e de estádios mais avançados (III e IV: 67,5\%). Não houve associação com metaplasia intestinal e/ou H. pylori. O índice apoptótico médio foi de 7,05 em 10 campos consecutivos de grande aumento e de 11,40 em 500 células (2,28\%). A positividade média ao PCNA foi de 275,05 células em 10 campos consecutivos de grande aumento e de 409,33 em 500 células $(81,9 \%)$. Houve correlação positiva do tipo histológico intestinal com PCNA e com a apoptose em 10 campos consecutivos de grande aumento. Houve correlação positiva entre a apoptose e PCNA tumorais. A sobrevida média foi de 28,41 meses. A idade (acima de 63) e o índice apoptótico (acima de 7,05), em análise multivariada, mostraram correlação negativa com a sobrevida. Conclusões - O adenocarcinoma da cárdia ocorreu mais nos indivíduos do sexo masculino, com idade média de 61 anos, com predomínio do tipo difuso e estádios mais avançados. A sobrevivência ainda é baixa. Houve correlação positiva entre a apoptose e proliferação celular tumoral. Tanto a idade como a apoptose foram fatores prognósticos independentes no câncer da cárdia.

DESCRITORES - Proliferação celular. Apoptose. Cárdia. Neoplasias gástricas. Adenocarcinoma.

\section{INTRODUÇÃO}

O câncer gástrico ainda é uma importante causa de mortalidade no mundo, embora sua incidência esteja em declínio nas últimas décadas. Representa a terceira neoplasia maligna mais freqüente no mundo e incide duas vezes mais em homens do que em mulheres. Cerca de dois terços dos casos ocorrem em países em desenvolvimento ${ }^{(27)}$.

No Brasil, segundo dados do Instituto Nacional do Câncer (INCA) de $2005^{(11)}$, representa o terceiro tipo de câncer mais letal nos homens e o quinto nas mulheres, com previsão de 11.145 óbitos, excluindo-se o câncer de pele não-melanoma. Nessa estatística, perde apenas para o câncer de mama feminina, pulmão, próstata, cólon e colo uterino.
No resto do mundo, dados estatísticos revelam maior incidência nos países como o Japão, China, países da América Central e Sul, Leste da Europa e, em menor grau, nos países como América do Norte, Norte da Europa, Austrália, Nova Zelândia e Índia(36).

Nas últimas décadas, tem ocorrido declínio na incidência do câncer gástrico, particularmente do terço distal do estômago, o que pode ser explicado pela redução na prevalência de fatores de risco, tais como mudança de hábitos alimentares, com aumento da ingesta de alimentos ricos em vitaminas $\mathrm{A}, \mathrm{C}$ e E, como frutas e verduras frescas, concomitante à diminuição do consumo de sal e tratamento da gastrite crônica pelo Helicobacter pylori ${ }^{(38)}$.

\footnotetext{
${ }^{1}$ Departamento de Patologia; ${ }^{2}$ Disciplina de Gastroenterologia Cirúrgica da Escola Paulista de Medicina - Universidade Federal de São Paulo, SP. Endereço para correspondência: Dra. Ana Maria A. A. Mader - Rua França Pinto, 832 - apt. 41 - Vila Mariana - 04016-003 - São Paulo, SP. E-mail: anamader@uol.com.br
} 
Por outro lado, particularmente no mundo ocidental, houve aumento da incidência do câncer do terço proximal do estômago. Alguns dados mostram prevalência maior no sexo masculino, forte associação com hérnia de hiato, refluxo gastroesofágico, esôfago de Barrett, obesidade, elevada classe social, etilismo e tabagismo ${ }^{(15)}$

Ainda quanto à origem, acredita-se que o H. pylori tenha menor importância na sua etiopatogênese, quando comparado ao carcinoma do terço distal do estômago. Há evidências de que essa bactéria possa exercer fator protetor no carcinoma da cárdia, talvez pela menor produção de ácido, que ocorre em indivíduos com tempo prolongado de infecção e, conseqüentemente, proteção ao refluxo gastroesofágico ${ }^{(2)}$.

Trata-se de neoplasia de comportamento mais agressivo, com prognóstico aparentemente pior que os tumores de terço distal, menor taxa de ressecabilidade, sendo que a sobrevivência global varia de $5 \%$ a $15 \%$ em 5 anos $^{(15)}$.

Em 1987, foi proposta uma classificação topográfica para os tumores da junção gastroesofágica. Definiu-se que câncer da junção esôfago-gástrica é aquele localizado desde $5 \mathrm{~cm}$ acima da transição esôfago-gástrica até $5 \mathrm{~cm}$ abaixo. O tipo I é o câncer do esôfago distal, o tipo II é o câncer da cárdia verdadeira e o tipo III, o subcárdico ${ }^{(31)}$.

Como em outras neoplasias, o prognóstico do câncer gástrico está diretamente ligado ao seu estádio evolutivo, sendo pior, obviamente, nas fases mais avançadas. A infiltração tumoral na parede gástrica, o comprometimento peritonial e das margens de ressecção cirúrgica, a propagação linfática regional, o tamanho do tumor e o grau de diferenciação histológica constituem fatores relacionados com o prognóstico e aceitos, universalmente, como estádio cirúrgico ${ }^{(4)}$. No entanto, destacam-se ainda alterações histopatológicas e de cinética celular, que estariam relacionados ao prognóstico desses doentes ${ }^{(8)}$.

\section{Objetivos}

Tendo em vista o aumento na incidência do câncer da cárdia nas últimas décadas, este trabalho visou estudar os aspectos clínico-patológicos, de proliferação celular e de apoptose no adenocarcinoma da cárdia, e suas eventuais influências no prognóstico destes doentes.

\section{CASUÍSTICA E MÉTODOS}

\section{Casuística}

No período entre janeiro de 1988 e dezembro de 2001, 75 pacientes foram submetidos a gastrectomia total por adenocarcinoma, na Disciplina de Gastroenterologia Cirúrgica do Hospital São Paulo da Universidade Federal de São Paulo-Escola Paulista de Medicina (UNIFESP-EPM). Destes, 40 casos em topografia da cárdia puderam ser analisados, retrospectivamente.

Este trabalho foi aprovado pelo Comitê de Ética em Pesquisa da UNIFESP-Hospital São Paulo, em 27 de setembro de 2002, registrado sob o número 0809/02.

Discriminou-se idade, sexo, recidiva local, evolução, levandose em consideração a presença ou ausência de metástase ao diagnóstico e tempo de sobrevivência.
Foram considerados critérios de exclusão para o estudo: neoplasia precoce e doentes com tratamento radio e/ou quimioterápico pré-operatório. Foram considerados fatores de exclusão para análise da sobrevivência: doentes com metástase à distância ao diagnóstico, com morte perioperatória e aqueles que faleceram de outras causas não relacionadas ao câncer gástrico.

\section{Métodos}

\section{Tipo de cirurgia}

Todos os tumores eram cárdicos ou subcárdicos, não sendo abordados aqueles de terço esofágico inferior. A cirurgia realizada foi a gastrectomia total, omentectomia e linfadenectomia D2, com reconstrução pela técnica de anastomose esôfago-jejunal em Y-de-Roux. A peça cirúrgica foi colocada em solução de formol a $10 \%$ e encaminhada para exame anatomopatológico.

\section{Anatomia patológica}

Ao exame macroscópico, não foi possível distinguir os tumores em tipo II ou III da classificação de Siewert, por serem todos avançados. Os fragmentos representativos foram incluídos em blocos de parafina, com cortes histológicos de $5 \mu \mathrm{m}$ e corados pela hematoxilina-eosina (H-E).

$\mathrm{Na}$ análise histopatológica, os tumores foram classificados segundo o tipo histológico de Laurén (intestinal ou difuso) e padrão de crescimento de Ming. Caso os tumores apresentassem os dois padrões histológicos (intestinal e difuso), optou-se pelo tipo predominante. Na mucosa adjacente ao tumor, analisou-se a presença ou ausência de metaplasia intestinal, de displasia epitelial e a pesquisa da bactéria $H$. pylori foi realizada pela coloração Giemsa modificada.

Para avaliação do grau de infiltração da neoplasia na parede gástrica, seguiu-se a classificação TNM, proposta pela União Internacional Contra o Câncer (American Joint Committee on Cancer, 1992). Embora houvesse uma versão atualizada datada de 2002, optou-se por usar a versão antiga, uma vez que, por se tratar de estudo retrospectivo, de acordo com os novos critérios, haveria um subestádio dos linfonodos. Todos os tumores eram avançados, ou seja, com comprometimento da túnica muscular própria, no mínimo. Para efeito de análise, a amostra foi subdividida em dois grupos: estádio menos avançado (I / II) e estádio mais avançado (III / IV).

Quanto ao comprometimento linfonodal, dividiu-se a amostra em dois grupos: ausência ou presença de metástase ganglionar. A relação entre o número de linfonodos comprometidos e o número total de linfonodos, multiplicado por 100 , foi denominada de percentual de linfonodos acometidos.

\section{Proliferação celular: PCNA}

O estudo imunoistoquímico foi realizado por meio da técnica estreptoavidina-biotina-peroxidase, utilizando-se o anticorpo anti-PCNA (clone PC10 Dako, código M0879), na diluição de 1:8000, em cortes histológicos de $4 \mu \mathrm{m}$ de espessura e em lâminas com silano. $\mathrm{O}$ controle positivo reagiu normalmente. Foi considerada positiva a célula cujo núcleo exibisse colorido castanho, independente da sua intensidade (Figura 1). Avaliou-se quantitativamente em 10 campos consecutivos de grande aumento (CGA), selecionando-se inicialmente, as áreas mais positivas 
sob o aumento de 100x e, posteriormente, até completar o total de 500 células neoplásicas avaliadas. Da relação entre as células neoplásicas positivas e negativas, multiplicada por 100, obteve-se o percentual de imunoexpressão de cada caso.

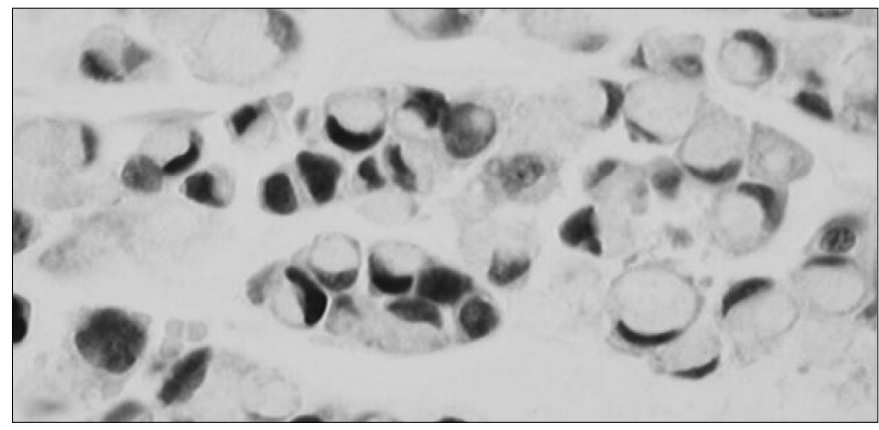

FIGURA 1 - Expressão nuclear do PCNA no adenocarcinoma difuso (imunoistoquímica PCNA - 400X)

Apoptose

A contagem dos corpúsculos apoptóticos foi feita em cortes histológicos corados ao H-E, em 10 CGA e em 500 células neoplásicas. Foram consideradas apenas as células claramente identificadas pelos critérios de KERR et al. ${ }^{(16)}$ : citoplasma eosinofílico e condensação cromatínica, presença de fragmentação nuclear, com ou sem formação de vesículas, na ausência de reação inflamatória ao redor (Figura 2). Caso houvesse dúvida em relação à morfologia, esta era confirmada sob o aumento de 1000x em imersão. Iniciou-se o estudo quantitativo nas áreas mais celulares e posteriormente, nos demais campos consecutivos.

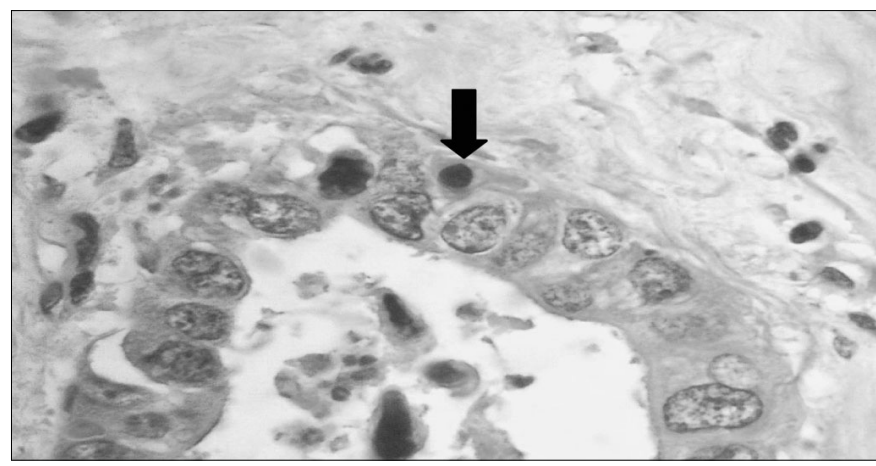

FIGURA 2 - Corpúsculo apoptótico no adenocarcinoma de tipo intestinal (H-E 1000X)

Análise quantitativa

Tanto a imunoexpressão do PCNA, como a contagem dos corpos apoptóticos, foram avaliadas quantitativamente, utilizandose um programa de análise de imagens equipado com o software Image-Pro Plus, versão 3.0, para Windows, da media Cybernetic. As imagens foram obtidas através de um microscópio Olympus Bx40 acoplado a uma videocâmara Sony CCD-IRIS, modelo DXC-107 e transferidas para um computador (Pentium $233 \mathrm{Mhz}$ ) com placa digitalizadora de imagens.

\section{Evolução dos doentes}

O acompanhamento ambulatorial dos doentes era semestral, com consulta clínica, solicitação de endoscopia digestiva alta, ultra-sonografia de abdome total ou tomografia computadorizada para identificação de recidiva. O menor tempo de avaliação da sobrevivência foi de 3 anos.

\section{Análise estatística}

Para as comparações entre as variáveis foram utilizados testes $t$ de Student e de Mann-Whitney. Para a correlação entre as variáveis, utilizou-se o índice de correlação de Pearson. Para o tempo de sobrevivência, foram construídas curvas de Kaplan-Meier e realizados testes de Log-rank e Breslow. Em relação às variáveis quantitativas, optou-se como valor referencial a mediana, no sentido de se homogeneizar as amostras a serem comparadas.

O Modelo de Regressão de Cox foi utilizado para analisar conjuntamente os efeitos das variáveis na sobrevida. O programa estatístico utilizado foi o SPSS versão 11.0 e o nível de significância adotado foi menor a 0,05 .

\section{RESULTADOS}

Dos 40 casos, as margens de ressecção cirúrgica estavam comprometidas no segmento proximal em quatro casos e no segmento distal em três. Havia metástase à distância ao diagnóstico (M1) em cinco casos.

Houve predomínio do sexo masculino ( $72,5 \%$ dos casos) sobre o feminino $(27,5 \%)$. O doente mais jovem tinha 33 anos e o mais velho 82. A idade média foi de 61,08 anos, com mediana de 63 .

Houve predomínio do tipo histológico difuso de Laurén (55\%) sobre o tipo intestinal (45\%). O padrão de crescimento tumoral, segundo Ming, tipo infiltrativo $(72,5 \%)$ predominou sobre o tipo expansivo $(27,5 \%)$.

Houve predomínio de casos com metástase ganglionar (N1 e $\mathrm{N} 2$ ), correspondendo a 70\% sobre aqueles sem metástase (N0), representados por $30 \%$ dos casos. Para oslinfonodos acometidos por metástase, o percentual médio foi de $41 \%$, com valor mediano de 38\% linfonodos. Os estádios mais avançados (III e IV) predominaram sobre os menos avançados (I e II).

$\mathrm{Na}$ mucosa adjacente ao tumor, a metaplasia intestinal esteve presente em 14 casos $(36,8 \%)$ e a pesquisa para bactéria $H$. pylori resultou positiva em 13 casos (34,2\%), sem haver correlação significativa entre estas variáveis, conforme mostra a Tabela 1. Não se detectou, em nenhum caso, displasia epitelial na mucosa adjacente.

TABELA 1 - Distribuição do carcinoma gástrico conforme a presença ou ausência de metaplasia intestinal e/ou $H$. pylori na mucosa adjacente ao tumor

\begin{tabular}{|c|c|c|c|c|c|}
\hline & & & \multicolumn{2}{|c|}{ Helicobacter pylori } & \multirow{2}{*}{ Total } \\
\hline & & & Sim & Não & \\
\hline \multirow{6}{*}{$\begin{array}{l}\text { Metaplasia } \\
\text { intestinal }\end{array}$} & $\operatorname{Sim}$ & $\mathrm{n}$ & 3 & 11 & 14 \\
\hline & & $\%$ linha & $21,4 \%$ & $78,6 \%$ & $100 \%$ \\
\hline & & $\%$ coluna & $23,1 \%$ & $44,0 \%$ & $36,8 \%$ \\
\hline & Não & $\mathrm{n}$ & 10 & 14 & 24 \\
\hline & & $\%$ linha & $41,7 \%$ & $58,3 \%$ & $100,0 \%$ \\
\hline & & $\%$ coluna & $76,9 \%$ & $56,0 \%$ & $63,2 \%$ \\
\hline \multirow[t]{3}{*}{ Total } & & $\mathrm{n}$ & 13 & 25 & 38 \\
\hline & & $\%$ linha & $34,2 \%$ & $65,8 \%$ & $100,0 \%$ \\
\hline & & $\%$ coluna & $100,0 \%$ & $100,0 \%$ & $100,0 \%$ \\
\hline
\end{tabular}

Teste exato de Fisher: $P=0,294$ 
O número médio de células neoplásicas positivas para o PCNA foi de 275,05 (10 CGA), e de 409,33 (500 células), perfazendo $81,86 \%$. O índice apoptótico médio foi de 7,05 (10 CGA) e de 11,40 (500 células), perfazendo 2,28\%. Houve correlação positiva entre o índice de PCNA e de apoptose, avaliados em 10 CGA $(P=0,0001)$. O índice apoptótico e de PCNA em 10 CGA, mostraram correlação positiva com os tumores de tipo intestinal (Tabela 2).

TABELA 2 - Comparações entre as com o tipo histológico de Laurén

\begin{tabular}{lccc}
\hline & $\begin{array}{c}\text { Intestinal }(\mathrm{n}=18) \\
\text { média }(\mathrm{ep})\end{array}$ & $\begin{array}{c}\text { Difuso }(\mathrm{n}=22) \\
\text { média }(\mathrm{ep})\end{array}$ & $\begin{array}{c}\text { Teste } \mathrm{t} \\
(\boldsymbol{P} \text {-valor })\end{array}$ \\
\hline PCNA - 10 CGA & $321,11(30,51)$ & $237,36(24,91)$ & $\mathbf{0 , 0 3 8 ^ { * }}$ \\
PCNA - 500 cels. & $435,44(18,70)$ & $387,96(23,47)$ & 0,134 \\
Apoptose - 10 CGA & $9,00(1,09)$ & $5,45(0,88)$ & $0,015^{*}$ \\
Apoptose - 500 cels. & $12,50(1,16)$ & $10,50(1,19)$ & 0,243 \\
\hline
\end{tabular}

Quanto à sobrevivência, excluindo-se os cinco pacientes que apresentavam metástase à distância ao diagnóstico, o tempo médio livre de óbito foi de 28,41 meses. Em análise univariada, obteve-se resultados significativos apenas para idade, ou seja, quanto maior a idade do paciente com carcinoma gástrico, pior a sua sobrevida ( $P=0,0367$ - Gráfico 1). Embora não houvesse resultado significativo, houve tendência para as variáveis metástase linfonodal $(P=0,062)$ e estádio $(P=0,0604)$, como mostra a Tabela 3 .

Ainda em análise univariada, também não se obteve resultado significativo para as variáveis PCNA e apoptose, em 10 CGA e em
500 células, embora houvesse tendência para a apoptose quando avaliada em 10 CGA $(P<0,2)$, como indica a Tabela 4 .

Em análise multivariada, as variáveis consideradas significantes foram a idade $(P=0,033)$ e apoptose em 10 CGA $(P=0,022)$, como mostra a Tabela 5.

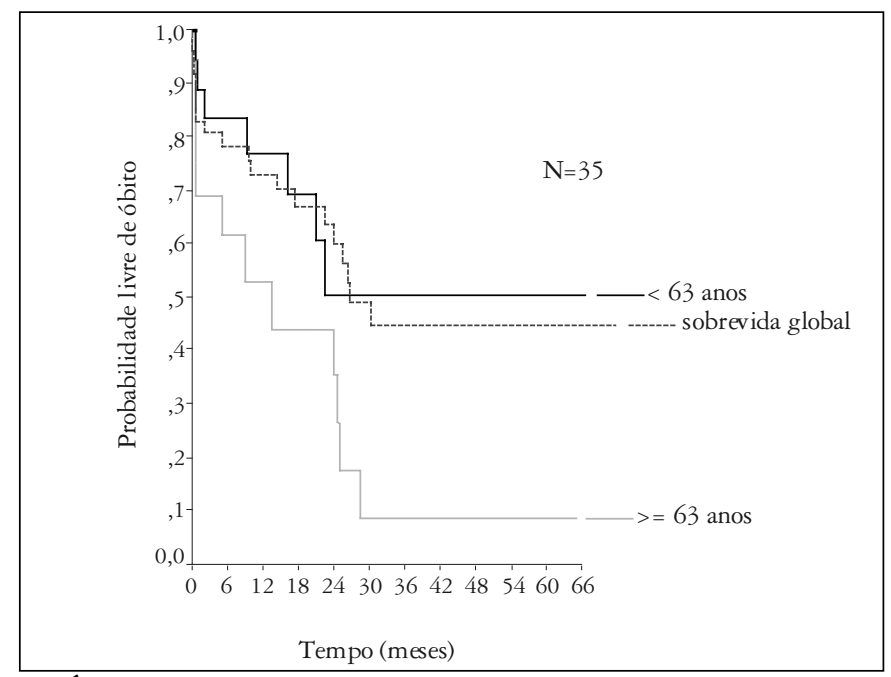

GRÁFICO 1 - Curva de sobrevivência global e segundo a idade para os doentes com adenocarcinoma gástrico (análise univariada - curva de Kaplan-Meier)

TABELA 3 - Sobrevivência dos doentes com carcinoma gástrico, segundo as variáveis metástase linfonodal e estadiamento

\begin{tabular}{|c|c|c|c|c|c|c|}
\hline Variáveis & Número de casos (n) & Tempo Médio livre de óbito* & I.C. $(95 \%)$ & Eventos & Censuras & $P$-valor** \\
\hline Metástase ausente & Ausente $(\mathrm{n}=12)$ & 40,46 & {$[23,74 ; 57,19]$} & $5(41,7 \%)$ & $5(41,7 \%)$ & 0,0621 \\
\hline Metástase presente & $\mathrm{N} 1$ ou N2 $(\mathrm{n}=23)$ & 20,98 & {$[10,22 ; 31,73]$} & $14(60,9 \%)$ & $9(39,1 \%)$ & \\
\hline Estádio menos avançado & I ou II $(n=13)$ & 40,46 & {$[23,74 ; 57,19]$} & $5(38,5 \%)$ & $8(61,5 \%)$ & 0,0604 \\
\hline Estádio mais avançado & III ou IV $(n=22)$ & 20,93 & {$[10,19 ; 31,68]$} & $14(63,6 \%)$ & $8(36,4 \%)$ & \\
\hline
\end{tabular}

TABELA 4 - Sobrevivência dos doentes com carcinoma gástrico, segundo a variável PCNA e apoptose, avaliada em 10 CGA e em 500 células

\begin{tabular}{|c|c|c|c|c|c|c|}
\hline Variável & Número de Casos (n) & Tempo livre de óbito * & I.C. $(95 \%)$ & Eventos & Censuras & $P$-valor ** \\
\hline \multirow[t]{2}{*}{ PCNA 10 CGA } & Até $283(\mathrm{n}=18)$ & 27,86 & {$[14,72 ; 41,00]$} & $11(61,1 \%)$ & $7(38,9 \%)$ & 0,9305 \\
\hline & Mais de $283(\mathrm{n}=17)$ & 27,82 & {$[12,86 ; 42,79]$} & $8(47,1 \%)$ & $9(52,9 \%)$ & \\
\hline \multirow[t]{2}{*}{ PCNA 500 cels. } & Até $459(\mathrm{n}=19)$ & 26,09 & {$[13,37 ; 38,81]$} & $12(63,2 \%)$ & $7(36,8 \%)$ & 0,5792 \\
\hline & Mais de $459(\mathrm{n}=16)$ & 30,31 & {$[14,67 ; 45,95]$} & $7(43,7 \%)$ & $9(56,3 \%)$ & \\
\hline \multirow[t]{2}{*}{ Apoptose 10 CGA } & Até $7(\mathrm{n}=21)$ & 33,50 & {$[20,64 ; 46,35]$} & $10(47,6 \%)$ & $11(52,4 \%)$ & 0,1203 \\
\hline & Acima de $7(n=14)$ & 19,02 & {$[5,74 ; 32,31]$} & $9(64,3 \%)$ & $5(35,7 \%)$ & \\
\hline \multirow[t]{2}{*}{ Apoptose 500 cels. } & Até 11 ( $\mathrm{n}=21)$ & 26,33 & {$[14,54 ; 38,11]$} & $12(57,1 \%)$ & $9(42,9 \%)$ & 0,7988 \\
\hline & Acima de $11(n=14)$ & 31,05 & {$[14,66 ; 47,45]$} & $7(50 \%)$ & $7(50 \%)$ & \\
\hline
\end{tabular}
* estimado pelo método de Kaplan-Meier

TABELA 5 - Análise multivariada de Cox para as variáveis idade, estádio avançado, proporção de linfonodos acometidos e apoptose em 10 CGA

\begin{tabular}{|c|c|c|c|c|}
\hline Variável & p-valor & Estimativa de risco relativo * & \multicolumn{2}{|c|}{ I.C. $95 \%$} \\
\hline Idade & $0,033^{*}$ & 1,064 & 1,005 & 1,126 \\
\hline Estadio III ou IV & 0,497 & 1,678 & 0,377 & 7,471 \\
\hline Proporção de linfonodos com metástase & 0,421 & 1,663 & 0,482 & 5,740 \\
\hline Apoptose em 10 CGA & $0,022^{*}$ & 1,142 & 1,019 & 1,280 \\
\hline
\end{tabular}




\section{DISCUSSÃO}

Trata-se de estudo retrospectivo com objetivo de analisar aspectos clínico-patológicos de proliferação celular e apoptose em 40 casos de carcinoma gástrico da cárdia. Outros estudos, considerando a topografia da cárdia, compreendem casuística similar ou pouco superior a presente, como o de LARDENOYE et al. ${ }^{(19)}$, com 49 pacientes, e o de ERTURK et al. ${ }^{(7)}$, com 60.

A análise da apoptose por meio da contagem dos corpúsculos apoptóticos à microscopia óptica, com a coloração H-E, tem sido adotada por diversos autores, desde quando os critérios morfológicos foram descritos por KERR et al. ${ }^{(16)}$ até os dias atuais, sendo considerado método bastante fidedigno e reprodutível ${ }^{(8)}$

Para a análise da sobrevivência (tempo médio livre de óbito), optou-se por excluir apenas os doentes que apresentassem metástase à distância ao diagnóstico ${ }^{(29)}$ e os com morte durante o ato cirúrgico ${ }^{(25)}$, fato este ausente nos casos deste estudo. Também não se excluíram os pacientes que tiveram margens de ressecção cirúrgica comprometidas microscopicamente porque, embora a literatura refira menor tempo de sobrevivência nesses casos, margem comprometida é considerada como doença residual, com risco de recidiva local. PAPACHRISTOU et al. ${ }^{(26)}$ consideram a recidiva da neoplasia o evento mais importante nos tumores em estádios iniciais (I e II), particularmente da cárdia, onde a cirurgia é intencionalmente curativa, pois pacientes em estádios mais avançados, como os desta casuística, geralmente morrem em decorrência das metástases, antes da recurrência do tumor na anastomose cirúrgica. Neoplasia microscópica residual, principalmente em margem proximal, tem sido relatada em percentual elevado nos pacientes operados por carcinoma da cárdia, sendo de $23 \%$ a $31,3 \% \%^{(1,13)}$.

Obteve-se predomínio no sexo masculino $(72,5 \%)$ em relação ao feminino $(27,5 \%)$, com idade média de 61,08 anos. Percentual muito semelhante foi encontrado em outros estudos retrospectivos clínico-patológicos similares, com carcinoma do terço proximal gástrico, com idade média variando de 57,8 a 69,7 anos ${ }^{(7,19)}$.

Esta casuística teve número pouco maior do tipo histológico difuso $(55 \%)$ sobre o intestinal $(45 \%)$, com predomínio do padrão de crescimento infiltrativo $(72,5 \%)$ sobre o expansivo. Outros trabalhos mostram predomínio ora do difuso ${ }^{(17)}$, ora do intestinal $^{(1,13)}$, e até mesmo distribuição equivalente ${ }^{(7)}$. Nesses estudos não houve especificação do padrão de crescimento tumoral de Ming, muito embora, de modo geral, exista associação entre o tipo intestinal e expansivo, assim como entre o tipo difuso e o infiltrativo ${ }^{(3)}$. Essa associação foi também comprovada no presente estudo.

Houve maior número de casos em estádios mais avançados da doença (67,5\% em estádios III e IV). Esses resultados estão de acordo com os de BRUNO et al.(1) que, ao compararem carcinomas da cárdia, antro e de coto gástrico, tiveram maior percentagem de casos em estádio mais avançado (T3 - 68,8\%) para o primeiro tipo. ERTURK et al. ${ }^{(7)}$ notaram predominância de tumores infiltrando até a serosa (T3), com linfonodos acometidos $(82 \%)$ e, portanto, em estádio mais avançado (III - 64\%).
Quanto à mucosa adjacente ao tumor, não se encontraram correlações significativas quanto à presença ou ausência da metaplasia intestinal e/ou do H. pylori, assim como também não se encontrou displasia epitelial associada em nenhum dos casos. De fato, a presença de gastrite crônica na mucosa adjacente, bem como dessa bactéria, está mais freqüentemente associada aos tumores gástricos não-cárdicos, em relação aos cárdicos e de esôfago distal, sendo que o índice de positividade para o H. pylori em tumores cárdicos, referido na literatura como sendo de $34 \%$, foi similar ao deste estudo ${ }^{(6)}$. A presença de metaplasia intestinal e/ou displasia também é considerada achado menos comum na mucosa adjacente aos carcinomas da cárdia, quando comparado aos adenocarcinomas originários em esôfago de Barrett ${ }^{(9)}$. Por terem sido analisados somente os tumores cárdicos e subcárdicos, o resultado desta série está de acordo com SIEWERT et al. ${ }^{(32)}$, que referem a metaplasia intestinal como sendo evento mais associado ao carcinoma da cárdia tipo I, infreqüente no tipo II e raro no tipo III.

O índice apoptótico foi de 7,05 (10 CGA) e de 11,40 (2,28\%), em 500 células. Esse índice foi maior no tipo intestinal $(9,00)$ em relação ao difuso $(5,45)$. Houve forte correlação entre o PCNA e a apoptose. Essa associação também foi descrita em outras neoplasias malignas, tais como de mama ${ }^{(28)}$, ovário ${ }^{(40)}$ e também em carcinoma gástrico ${ }^{(10,14,18)}$. Índices apoptóticos similares aos da presente casuística foram descritos como em $2,05 \%{ }^{(8,10)}$.

$\mathrm{Na}$ literatura, encontrou-se índice apoptótico médio pouco menor que o deste estudo, referido em $1,58 \%^{(12,18)}$ e maiores, sendo de $8,1 \%$ nos carcinomas mais diferenciados (grau 1 e grau 2 ) e de $4,7 \%$ naqueles pouco diferenciados (grau 3$)^{(14,34)}$.

O índice médio PCNA nesta série em 500 células, foi de $81,86 \%$, maior do que aquele obtido por outros autores, que variaram de $23 \%$ a $49 \%{ }^{(6,12,23,35)}$.

Neste estudo, o tempo médio livre de óbito foi de 28,41 meses. Tempo médio de sobrevivência similar ao desta série foi encontrado em doentes operados por carcinoma da cárdia ${ }^{(7)}$ ou pouco inferiores como de 23 meses ${ }^{(1,19)}$. DEMIRKAN et al. ${ }^{(3)}$, em estudo comparativo entre tumores proximais e distais gástricos, encontraram sobrevivência menor para os tumores de terço proximal (22,0 meses), em relação aos distais (40,0 meses).

Em análise univariada e multivariada, a idade acima de 63 anos mostrou correlação negativa significativa com o tempo médio livre de óbito. Diversos trabalhos têm mostrado o mesmo, ou seja, idade acima de 63 anos comportou-se como fator prognóstico independente ${ }^{(7,12,13)}$.

A presença de metástase linfonodal e o estádio (I/II e III/IV), ambos relacionados entre si, não tiveram correlação significativa com o tempo livre de óbito. No entanto, na análise univariada, houve tendência para os dois primeiros parâmetros $(P=0,0621$ e 0,0604 , respectivamente), resultado esse que, provavelmente, seria significativo se a presente casuística fosse maior. Essas correlações já estão bem estabelecidas na literatura, com diversos trabalhos mostrando a importância do estádio no prognóstico do câncer gástrico ${ }^{(3,4,13)}$.

Quanto à proliferação celular tumoral, não se encontrou correlação entre o PCNA e o tempo livre de óbito, quando 
analisado em 10 CGA ou em 500 células. O mesmo resultado foi observado em estudos em carcinoma gástrico ${ }^{(24,30)}$. Ao contrário, MAEDA et al. ${ }^{(22,23)} \mathrm{e}$ ELPEK et al. ${ }^{(6)}$ encontraram pior prognóstico para os casos em que os tumores tinham elevado índice de proliferação celular.

Com relação à apoptose, embora não fosse encontrada correlação, em análise univariada, entre essa e o tempo de sobrevivência, o resultado foi significativo em análise multivariada. Diversos estudos têm mostrado a correlação positiva entre a apoptose e a proliferação celular e que elevado índice apoptótico associa-se com piora do prognóstico em vários tipos de carcinomas, como de mama ${ }^{(21)}$, estômago ${ }^{(10)}$, cólon $^{(33)}$ e pulmão ${ }^{(37)}$.

Em carcinomas gástricos, estudos da apoptose por meio de suas proteínas indutoras, como a FasL e Caspase-3, mostraram correlação positiva entre a expressão das mesmas com o tamanho da neoplasia e a presença de metástase linfonodal ${ }^{(12,39)} \mathrm{e}$, conseqüentemente, pior prognóstico. Outros trabalhos, envolvendo proteína inibidora da apoptose, como o Bcl-2, mostraram correlação negativa entre a expressão da proteína com o índice apoptótico, com conseqüente melhor sobrevivência dos doentes ${ }^{(20)}$.

\section{CONCLUSÕES}

O adenocarcinoma gástrico da cárdia ocorreu preferencialmente em indivíduos do sexo masculino, com idade média de 61 anos, com predomínio do tipo histológico difuso, infiltrativo e em estádios mais avançados da doença. Houve correlação positiva entre a apoptose e a proliferação celular tumoral. A sobrevivência dos doentes ainda é baixa, sendo de 28,41 meses em média. Tanto a idade como a apoptose foram fatores prognósticos independentes no adenocarcinoma da cárdia.

\section{AGRADECIMENTOS}

À Ângela Tavares Paes, estatística da Disciplina de Bioestatística do Departamento de Medicina Preventiva da UNIFESP-EPM, pela primorosa análise estatística deste estudo.

Mader AMAA, Patrício FRS, Rigueiro MP, Lourenço LG. Analysis of clinicopathological, tumor cell proliferation and apoptosis parameters in adenocarcinoma of the gastric cardia. Arq Gastroenterol. 2006;43(3): 184-90.

ABSTRACT - Background/Aims - In view of the increased incidence of carcinoma of the cardia over recent years, this work had the aim of studying the clinicopathological aspects, cell proliferative and tumor apoptotic indices of this neoplasm, their interrelations and possible influences on the prognosis. Material and Methods - Forty cases of adenocarcinoma of the cardia were studied between 1988 and 2001 , with a minimum clinical follow-up of 3 years. Patients were excluded if they had previous chemotherapy or radiotherapy treatment, presented early neoplasia, or died during the operations or for other reasons unrelated to cancer. Gender; age, Laurén and Ming histological type, staging, and the presence or absence of intestinal metaplasia, epithelial dysplasia and Helicobacter pylori in the adjacent mucosa were analyzed. The apoptotic index was evaluated via hematoxylin-eosin in the primary tumor. To analyze the cell proliferation tumor, PCNA was utilized. The immunohistochemical technique utilized was streptavidin-biotin-peroxidase. For the survival analysis, cases with distant metastasis upon diagnosis were excluded. For the statistical analysis, the Student $t$ and Mann-Whitney tests, Kaplan-Meier curves and Cox regression model were utilized. Results - The mean age was 61 years (median: 63). There was predominance of the male gender (72.5\%), diffuse histological type (55\%) and infiltrative histological type (72.5\%), and the more advanced stages (III and IV: 67.5\%). There was no association with intestinal metaplasia and/or H. pylori. No epithelial dysplasia was detected in adjacent mucosa in any of the cases. The mean apoptotic index was 7.05 in 10 high power fields and 11.40 in 500 cells (2.28\%). The mean positivity to PCNA was 275.05 cells in ten high power fields and 409.33 in 500 cells $(81.9 \%)$. There was a positive correlation between the cell proliferative and apoptotic indices. There was a positive correlation for intestinal histological type with PCNA and apoptotic indices, in 10 high power fields. The mean survival was 28.41 months. Age over 63 and apoptotic index over 7.05 showed a negative correlation with survival, in multivariate analysis. Conclusions - Adenocarcinoma of the cardia predominated in male adults of mean age 61 years, and the predominant type was diffuse in more advanced stages. There was a correlation between apoptosis and tumoral cell proliferation. Survival in cases of adenocarcinoma of the cardia is still low. Both age and apoptosis were independent prognostic factors in cancer of the cardia.

HEADINGS - Cell proliferation. Apoptosis. Cardia. Stomach neoplasms. Adenocarcinoma. 


\section{REFERÊNCIAS BIBLIOGRÁFICAS}

1. Bruno L, Nesi G, Montinaro F, Carassale G, Lassig R, Boddi V, Bechi P; Cortesini C. Clinicopathologic findings and results of surgical treatment in cardiac adenocarcinoma. J Surg Oncol. 2000;74:33-5.

2. Chow WH, Blaser MJ, Blot WJ, Gammon MD, Vaughan TL, Risch HA, Perez-Perez GI, Schoenberg JB, Stanford JL, Rotterdam H, West AB, Fraumeni JF. An inverse relation between $\mathrm{CagA}+$ strains of $\mathrm{H}$. pylori infection and risk of esophageal and gastric cardia adenocarcinoma. Cancer Res. 1998;58:588-90.

3. Demirkan NC, Tuncyürek, Ertan EU, Alkanat MB, Icöz G. Correlation of histological classifications of carcinomas with location and prognosis. Gastroenterol Clin Biol. 2002;26:610-5.

4. Dicken BJ, Saunders LD, Jhangri GS, de Gara C, Cass C, Andrews S, Hamilton SM Gastric cancer: establishing predictors of biologic behavior with use of populationbased data. Ann Surg Oncol. 2004;11:629-35.

5. Driessen A, Van Raemdonck D, De Leyn P, Filez L, Peeters M, Winnepenninckx V, Penninckx F, Lerut T, Ectors N, HP Belgian Contact Group. Are carcinomas of the cardia oesophageal or gastric adenocarcinomas? Eur J Cancer. 2003;39:2487-94.

6. Elpek GO, Gelen T, Aksoy NH, Karpuzoglu T, Keles N. Microvessel count, proliferating cell nuclear antigen and Ki-67 indices in gastric adenocarcinoma. Pathol Oncol Res. 2000;6:59-64.

7. Erturk MS, Cicek Y, Ersan Y, Saribeyoglu K, Dogusoy G, Erginoz E. Analysis of clinicopathological prognostic parameters in adenocarcinoma of the gastric cardia. Acta Chir Belg. 2003;103:611-5.

8. Forones NM, Carvalho AP, Giannotti-Filho O, Lourenço LG, Oshima CT. Cel proliferation and apoptosis in gastric cancer and intestinal metaplasia. Arq Gastroenterol. 2005;42:30-4

9. Goldblum JR. The significance and etiology of intestinal metaplasia of the esophagogastric junction. Ann Diagn Pathol. 2002:6:67-73.

10. Ikeguchi M, Cai J, Yamane N, Maeta M, Kaibara N. Clinical significance of spontaneous apoptosis in advanced gastric adenocarcinoma. Cancer. 1999;85:2329-35.

11. Instituto Nacional do Câncer - INCA. Estimativas da incidência da mortalidade por câncer no Brasil. Rio de Janeiro; 2005.

12. Isobe N, Onodera H, Mori A, Shimada Y, Yang W, Yasuda S, Fujimoto A, Ooe H, Ari $\mathrm{S}$, Kitaichi M, Imamura M. Caspase-3 expression in human gastric carcinoma and its clinical significance. Oncology. 2004;66:201-9.

13. Jakl RJ, Miholic J, Koller R, Markis E, Wolner E. Prognostic factors in adenocarcinoma of the cardia. Am J Surg. 1995;169:316-20.

14. Jesionek-Kupnicka D, Tenderenda M, Rutkowski P. Extent of spontaneous apoptosis in gastric cancer: relation to proliferative index, $\mathrm{p} 53$ expression, CD34 expression and histopathological features. J Exp Clin Cancer Res. 2002;21:371-5.

15. Kassab P, Malheiros CA. Câncer da cárdia. In: Gama-Rodrigues JJ, Lopasso FP, De Grande JC, Safatle NF, Bresciani C, Malheiros CA, editores. Câncer do estômago. Aspectos atuais do diagnóstico e tratamento. São Paulo: Andrei; 2002. p.241-51.

16. Kerr JFR, Wyllie AH, Currie AR. Apoptosis: a basic biological phenomenon with wide-ranging implications in tissue kinetics. Br J Cancer. 1972;26:239-57.

17. Kim JP, Kim YW, Yang HK, Noh DY. Significant prognostic factors by multivariate analysis of 3926 gastric cancer patients. World J Surg. 1994;18:872-7.

18. Koshida Y, Saegusa M, Okayasu I. Apoptosis, cell proliferation and expression of $\mathrm{Bcl}-2$ and Bax in gastric carcinomas: immunohistochemical and clinicopathological study. Br J Cancer. 1997;75:367-73.

19. Lardenoye LW, Kappetein P, Lagaay MB, Allema JH, Breslau PJ. Survival of proximal third gastric carcinoma. J Surg Oncol. 1998;68:183-6.

20. Lee HK, Lee HS, Yang HK, Kim WH, Lee KU, Choe KJ, Kim JP. Prognostic significance of Bcl-2 and p53 expression in gastric cancer. Int J Colorectal Dis. 2003;18:518-25.

21. Lipponen P, Papinaho S, Eskelinen M, Klemi PJ, Aaltomaa S, Kosma VM, Marin S, Syrjänen K. DNA ploidy, S-phase fraction and mitotic indices as prognostic predictors of female breast cancer. Anticancer Res. 1992;12:1533-8.
22. Maeda K, Chung YS, Onoda N, Kato Y, Nitta A, Arimoto Y, Yamada N, Kondo Y, Sowa M. Proliferating cell nuclear antigen labeling index of preoperative biopsy specimens in gastric carcinoma with special reference to prognosis. Cancer. 1994;73:528-33.

23. Maeda K, Chung YS, Takatsuka S, Ogawa Y, Onoda N, Sawada T, Kato Y, Nitta A, Arimoto $\mathrm{Y}$, Kondo $\mathrm{Y}$. Tumor angiogenesis and tumor cell proliferation as prognostic indicators in gastric carcinoma. Br J Cancer. 1995;72:319-23.

24. Mangham DC, Rowlands DC, Newbold KM, Reynolds GM, Fielding JW, Hallissey MT. Expression of proliferating cell nuclear antigen (PCNA) in gastric carcinoma: no evidence for prognostic value. J Clin Pathol. 1994;47:473-4.

25. Noda S, Soejima K, Inokuchi K. Clinicopathological analysis of the intestinal type and diffuse type of gastric carcinoma. Jpn J Surg. 1980;10:277-83.

26. Papachristou DN, Agnanti N, D'Agostino H, Fortner JG. Histologically positive esophageal margin in the surgical treatment of gastric cancer. Am J Surg. 1980;139:711-3.

27. Plummer M, Franceschi S, Munoz N. Epidemiology of gastric cancer. IARC Sci Publ. 2004;157:311-26.

28. Rasbridge SA, Gillett CE, Seymour AM, Patel K, Richards MA, Rubens RD, Millis RR. The effects of chemotherapy on morphology, cellular proliferation, apoptosis and oncoprotein expression in primary breast carcinoma. Br J Cancer. 1994;70:335-41.

29. Saito H, Tsujitani S, Kondo A, Ikeguchi M, Maeta M, Kaibara N. Expression of vascular endothelial growth factor correlates with hematogenous recurrence in gastric carcinoma. Surgery. 1999;125:195-201.

30. Setälä LP, Kosma VM, Marin S, Lipponen PK, Eskelinen MJ, Syrjänen KJ, Alhava EM. Prognostic factors in gastric cancer: the value of vascular invasion, mitotic rate and lymphoplasmacytic infiltration. Br J Cancer. 1996;74:766-72.

31. Siewert JR, Stein HJ. Carcinoma of the cardia: carcinoma of the gastroesophageal junction - classification, pathology and extent of resection. Dis Esophagus. 1996;9:173-82.

32. Siewert JR, Feith M, Stein HJ. Biologic and clinical variations of adenocarcinoma at the esophago-gastric junction: relevance of a topographic-anatomic subclassification. J Surg Oncol. 2005;90:139-46.

33. Sinicrope FA, Hart J, Hsu H, Lemoine M, Michelassi F, Stephens LC. Apoptotic and mitotic indices predict survival rates in lymph node-negative colon carcinomas. Clin Cancer Res. 1999;5:1793-804

34. Shinohara T, Ohshima K, Murayama H, Kikuchi M, Yamashita Y, Shirakusa T. Apoptosis and proliferation in gastric carcinoma: the association with histological type. Histopathology. 1996;29:123-9.

35. Tenderenda M, Rutkowski P, Jesionek-Kupnicka D, Kubiak R. Expression of CD34 in gastric cancer and its correlation with histology, stage, proliferation activity, p53 expression and apoptotic index. Pathol Oncol Res. 2001;7:129-34.

36. Terry MB, Gaudet MM, Gammon MD. The epidemiology of gastric cancer. Semin Radiat Oncol. 2002;12:111-27.

37. Törmänen U, Eerola AK, Rainio P, Vähäkangas K, Soini Y, Sormunen R, Bloigu R, Lehto VP, Pääkkö P. Enhanced apoptosis predicts shortened survival in non-small cell lung carcinoma. Cancer Res. 1995;55:5595-602.

38. Tsugane S. Salt, salted food intake, and risk of gastric cancer: epidemiologic evidence. Cancer Sci. 2005;96:1-6.

39. Zheng HC, Sun JM, Wei ZL, Yang XF, Zhang YC, Xin Y. Expression of Fas ligand and caspase- 3 contributes to formation of immune escape in gastric cancer. World $\mathrm{J}$ Gastroenterol. 2003;9:1415-20.

40. Yamasaki F, Tokunaga O, Sugimori H. Apoptotic index in ovarian carcinoma: correlation with clinicopathologic factors and prognosis. Gynecol Oncol. 1997;66:439-48. 